\title{
Lack of global meiotic sex chromosome inactivation, and paucity of tissue-specific gene expression on the Drosophila $X$ chromosome
}

\author{
Lyudmila M Mikhaylova ${ }^{1}$ and Dmitry I Nurminsky ${ }^{1,2^{*}}$
}

\begin{abstract}
Background: Paucity of male-biased genes on the Drosophila X chromosome is a well-established phenomenon, thought to be specifically linked to the role of these genes in reproduction and/or their expression in the meiotic male germline. In particular, meiotic sex chromosome inactivation (MSCl) has been widely considered a driving force behind depletion of spermatocyte-biased $X$-linked genes in Drosophila by analogy with mammals, even though the existence of global MCSI in Drosophila has not been proven.

Results: Microarray-based study and qRT-PCR analyses show that the dynamics of gene expression during testis development are very similar between $X$-linked and autosomal genes, with both showing transcriptional activation concomitant with meiosis. However, the genes showing at least ten-fold expression bias toward testis are significantly underrepresented on the $X$ chromosome. Intriguingly, the genes with similar expression bias toward tissues other than testis, even those not apparently associated with reproduction, are also strongly underrepresented on the $X$. Bioinformatics analysis shows that while tissue-specific genes often bind silencingassociated factors in embryonic and cultured cells, this trend is less prominent for the $X$-linked genes.

Conclusions: Our data show that the global meiotic inactivation of the $X$ chromosome does not occur in Drosophila. Paucity of testis-biased genes on the $X$ appears not to be linked to reproduction or germline-specific events, but rather reflects a general underrepresentation of tissue-biased genes on this chromosome. Our analyses suggest that the activation/repression switch mechanisms that probably orchestrate the highly-biased expression of tissue-specific genes are generally not efficient on the $X$ chromosome. This effect, probably caused by dosage compensation counteracting repression of the $X$-linked genes, may be the cause of the exodus of highly tissuebiased genes to the autosomes.
\end{abstract}

\section{Background}

Traffic of the newly-created testis-specific genes from the $X$ chromosome to autosomes via retroposition is a major trend conserved between different taxa [1-3]. In mammals, it is believed to be an adaptation to the evolutionarily 'young' meiotic sex chromosome inactivation (MSCI), so that the newly-duplicated autosomal genes 'rescue' the silencing of the parental $X$-linked genes in the male germline [4]. This process would lead to the loss of the $X$-linked genes that function specifically in the MSCI-affected male germline cells, namely

\footnotetext{
* Correspondence: dnurminsky@som.umaryland.edu

'Department of Anatomy and Cellular Biology, Tufts University School of Medicine, Boston, USA

Full list of author information is available at the end of the article
}

spermatocytes. Indeed, spermatocyte-specific genes are severely underrepresented on the $X$ chromosome in mice, illustrating the proposed role of MSCI in genome evolution. In contrast, the male-specific genes expressed at either earlier or later stages of spermatogenesis (that is in spermatogonia or in round spermatids) are overrepresented on the mouse $X$ chromosome [5,6], consistent with the vigorous selection for male-beneficial $X$ linked genes proposed previously [7].

Reports on the existence of MSCI as a mechanism of global inactivation of the $X$-linked genes in spermatocytes of Drosophila are not conclusive. In flies, similar to mammals, spermatocytes rapidly proceed through the S-phase and enter extended prophase I of meiosis, which lasts several days (reviewed in [8]). The

\section{(Ciomed Central}


premature cytological condensation of the $X$ chromosome in primary spermatocytes and the dominant male sterility frequently caused by $X$-autosomal translocations were originally put forward as indicators of MSCI in Drosophila [9]. The overall paucity of $X$-linked testisspecific genes and the 'exodus' of the newly-duplicated testis-specific genes from the $X$ chromosome via retroposition have been reported in flies and interpreted as additional evidence of MSCI [1,10-14]. However, a recent study indicates that retroposing genes avoid the $X$ regardless of their expression pattern, and probably owing to their intrinsic integration preferences [15]. The finding that the $X$-linked transgenes driven by a testisspecific ocn promoter fail to show active expression [16] further supported the MSCI model, even though the 'sampling' of chromosomes by the transgene insertions was not very comprehensive. Finally, microarray analyses of dissected testes showed lower $X$-linked gene expression in the sample enriched with spermatocytes as compared to samples that included earlier or later developmental stages [17]. However, this finding could have been affected by differences in the somatic cell content between different regions of testis because somatic cells are underrepresented in the spermatocyteenriched sample; therefore, the genes expressed in somatic cells, but not in germline, could be perceived as inactivated in spermatocytes. On the other hand, no significant dearth of $X$-linked transcription has been detected in another genome-wide analysis of gene expression in gonads, arguing against global inactivation of the $X$ chromosome in the meiotic germline [18]. However, active gene expression in spermatogonia and somatic testis cells [19] present in the whole adult testes could in principle 'mask' the putative gene silencing in meiotic cells in this study.

In order to understand better the genome evolution and structure, and to obtain further insight into the tissue-specific gene expression, it is important to determine whether the paucity of male-biased $X$-linked genes in flies is due to MSCI or other novel, yet unknown mechanism(s). To determine whether the expression of $X$-linked genes changes concomitant with meiosis, we performed a study of the testis transcriptome in larval development, tracing the maturation of the first wave of spermatocytes through the third instar and up to the meiotic divisions at the beginning of pupation [20]. In this model, the 'load' of spermatogonia and associated somatic cells is similar between the developmental stages; therefore the differences in gene expression are attributable to an increase in the number of spermatocytes and to their maturation. While the expression of testis-specific $X$-linked genes steadily increased during spermatocyte maturation as expected, global gene expression on the $X$ chromosome did not significantly differ from the autosomes throughout the entire period - both results being consistent with the lack of global MSCI. Further, we found that the paucity of testisbiased genes is not a unique example and that other tissue-biased genes are also underrepresented on the $X$ chromosome, indicative of this trend not being limited to the reproduction-related genes. Our analyses suggest that the paucity of $X$-linked tissue-specific genes may be driven by the general inefficiency of stringent tissue-specific gene regulation on the $X$ chromosome.

\section{Results and Discussion}

$X$-linked and autosomal testis-biased genes show similar longitudinal profiles of expression in testis development MSCI should lead to a paucity of the spermatocyte-specific genes on the $X$ chromosome, as has been shown in mammals [5]. In Drosophila, the vast majority of the testis-specific genes analyzed to date are activated in primary spermatocytes, that is in the meiotic prophase I [21]. To determine whether expression profiles of $X$ linked genes deviate from this trend, we quantitatively analyzed the transcription of eight previously characterized testis-biased $X$-linked genes in larval testis development, using real-time RT-PCR. In parallel, 18 autosomal testis-biased genes were analyzed as a reference group (Table 1) [22-25].

Table 1 The $X$-linked and autosomal spermatocytespecific genes analyzed for their temporal expression during testis development

\begin{tabular}{lccc}
\hline Gene & Autosomal & X-linked & Reference \\
\hline Sdic & & + & {$[22]$} \\
CG15450 & & + & {$[12]$} \\
CG1314 & & + & {$[12]$} \\
CG1338 & & + & {$[12]$} \\
CG15711 & & + & {$[12]$} \\
CG15452 & & + & {$[12]$} \\
CG11227 & & + & {$[12]$} \\
CG1324 & & & {$[12]$} \\
Dj & & & {$[23]$} \\
fZO & + & & {$[24]$} \\
$\beta$ (2)Tubulin & + & & {$[25]$} \\
OCn & + & & {$[16]$} \\
CG6262 & + & & {$[12]$} \\
CG3483 & + & & {$[12]$} \\
CG7813 & + & & {$[12]$} \\
CG15874 & + & & {$[12]$} \\
CG3494 & + & & {$[12]$} \\
CG16837 & + & & {$[12]$} \\
CG4439 & + & & {$[12]$} \\
CG4750 & + & & {$[12]$} \\
CG15873 & + & & \\
CG15925 & + & & \\
\hline & + & & \\
\hline
\end{tabular}


Testes were collected daily from day four (second instar) through day seven (Figure 1, dL), at which point third-instar larvae started to pupate (Figure 1, dP). Since the first wave of germline differentiation reaches meiotic divisions approximately at the onset of pupation [19] and spermatocytes become the predominant cell type in testes of third instar larvae [26], this analysis traced changes in gene expression associated with the accumulation and maturation of spermatocytes. Accordingly, transcripts of known spermatocyte-specific genes, both autosomal and $X$-linked, showed a steady increase throughout testis development in our series of samples (dotted lines on Figure 1A, B). Other analyzed genes, including those located on the $X$ chromosome, showed the same pattern indicating that their activation occurs in the meiotic germline. The pooled data also show a striking similarity of the expression patterns between the groups of $X$-linked and autosomal genes (Figure $1 C)$. We further sought to determine whether the relatively small selection of $X$-linked genes for our study could have influenced our conclusion that these genes are usually activated concomitant with meiosis. To assess a larger sample, we analyzed published in situ hybridization data for $501 X$-linked genes [21] and found that 474 of them $(94 \%)$ are induced in primary spermatocytes. Cumulatively, the above data obtained by different approaches convincingly show that numerous $X$-linked genes are activated in the male meiotic germline. It is not clear then why the transgenes driven by promoter of the testis-specific ocn gene could not show efficient expression when integrated into the $X$ chromosome [16], given that ocn shows the same expression profile as endogenous $X$-linked testis-biased genes (compare the solid line on Figure $1 \mathrm{~B}$ for ocn to the profiles in Figure 1A). However, regardless of the mechanism(s) of silencing, the ocn transgenes [16] do not appear representative of the $X$-linked genes that are commonly expressed in spermatocytes.

\section{Global gene expression on the $X$ chromosome does not significantly change during testis development}

To further support our findings by extending them to the majority of $X$-linked genes, we analyzed the global trends of $X$-linked gene expression during testis development using gene microarrays. To improve the resolution of the analysis, we cultured larvae at a lower temperature $\left(18^{\circ} \mathrm{C}\right)$ and collected testes from feeding larvae at fourth, fifth, sixth and seventh days of development (Figure $2 \mathrm{dL}_{\mathrm{f}}$ ), and from wandering larvae at days seven and ten, just before the onset of pupation at days 11 to 12 (Figure 2, $\mathrm{dL}_{\mathrm{w}}$ ). Throughout larval testis development, we did not find appreciable differences in expression between the $X$ chromosome and autosomes (Figure 2, orange versus blue bars) or any substantial reduction in $X$-linked gene expression (Figure 2, orange bars), strengthening the hypothesis that the $X$

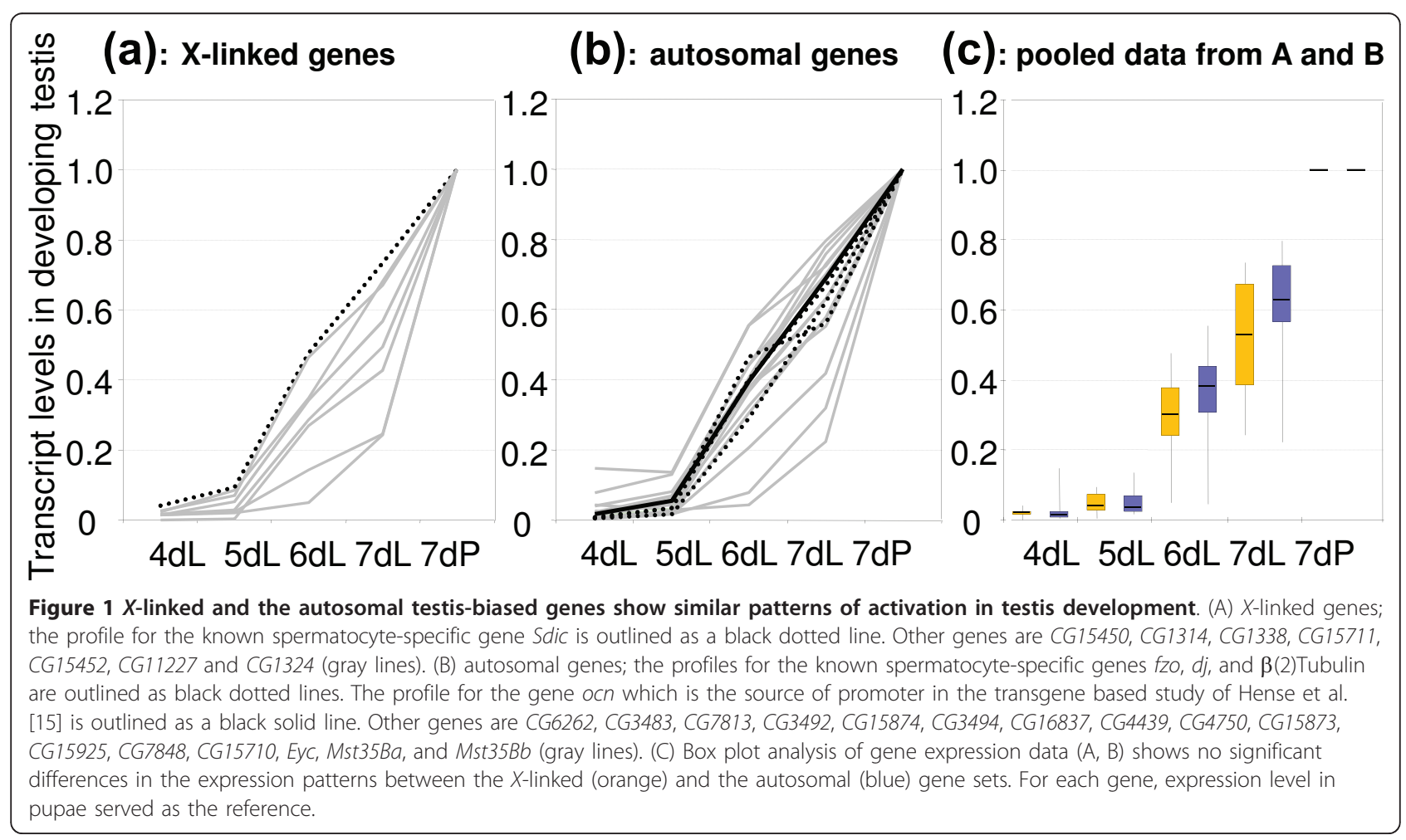




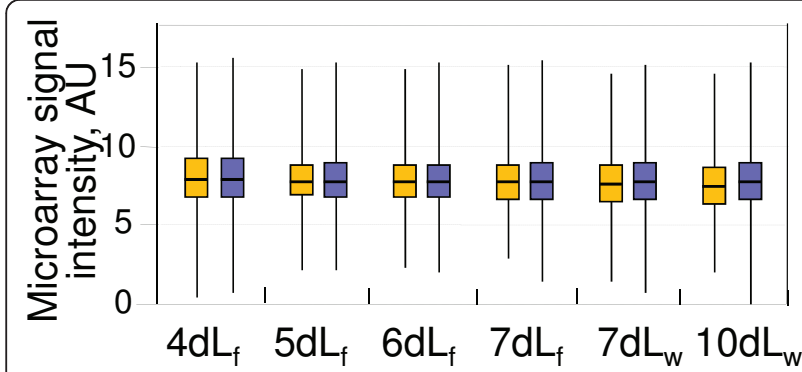

Figure 2 Lack of global $X$-chromosome inactivation in developing testes. Expression of $X$-linked (orange) and autosomal (blue) genes was measured as signal intensity of corresponding microarray probes, after normalization. cDNAs hybridized to the microarrays were isolated from testes of either feeding $(f)$ or wandering $(w)$ larvae grown for the indicated number of days at $18^{\circ} \mathrm{C}$. The analysis traces the first wave of germline differentiation; pupation indicative of the meiotic divisions occurred at day 11 .

chromosome is not affected by global MSCI in Drosophila, contrary to mammalian spermatogenesis.

Weak testis bias of expression shown by $X$-linked genes Although we did observe numerous $X$-linked genes increasing their expression during testis development, previous reports showed a paucity of male-biased genes on the $X$ [12-14]. To define the relationship between these two gene categories, we analyzed expression bias of the genes up-regulated in developing testes. Using published gene expression data [27], we compared microarray signals observed with adult testis-derived RNA to the signals obtained with RNA from a variety of somatic sources including accessory glands, brain, head, thoracicoabdominal ganglion, crop, midgut, hindgut, malpigian tubules of adults and larvae, ovary, salivary glands, and carcass. Conservatively, the minimal testis-to-somatic tissue signal ratio across the entire panel of analyzed samples was defined as a measure of bias of gene expression toward testis. This analysis identified about $50 \%$ of the autosomal genes up-regulated in testis development as highly testis-biased (minimal testis::somatic tissue signal ratio $\geq 10$ ) (Figure 3, blue bars). Strikingly, highly-biased genes constitute less than one-quarter of the $X$-linked genes, and this difference from the autosomal genes is highly significant $\left(\chi^{2}\right.$ test, $P=3 \times 10^{-10}$ ) (Figure 3 , orange bars). Apparently, it is the cohort of highly testis-biased genes that is depleted in the pool of the $X$-linked genes up-regulated in testis development. As a result, on average the $X$-linked genes show a weaker testis bias of expression than their autosomal counterparts.

Diverse tissue-specific genes are underrepresented on the $X$ chromosome

Our gene expression study on developing testes indicated that the paucity of testis-biased genes on the $X$

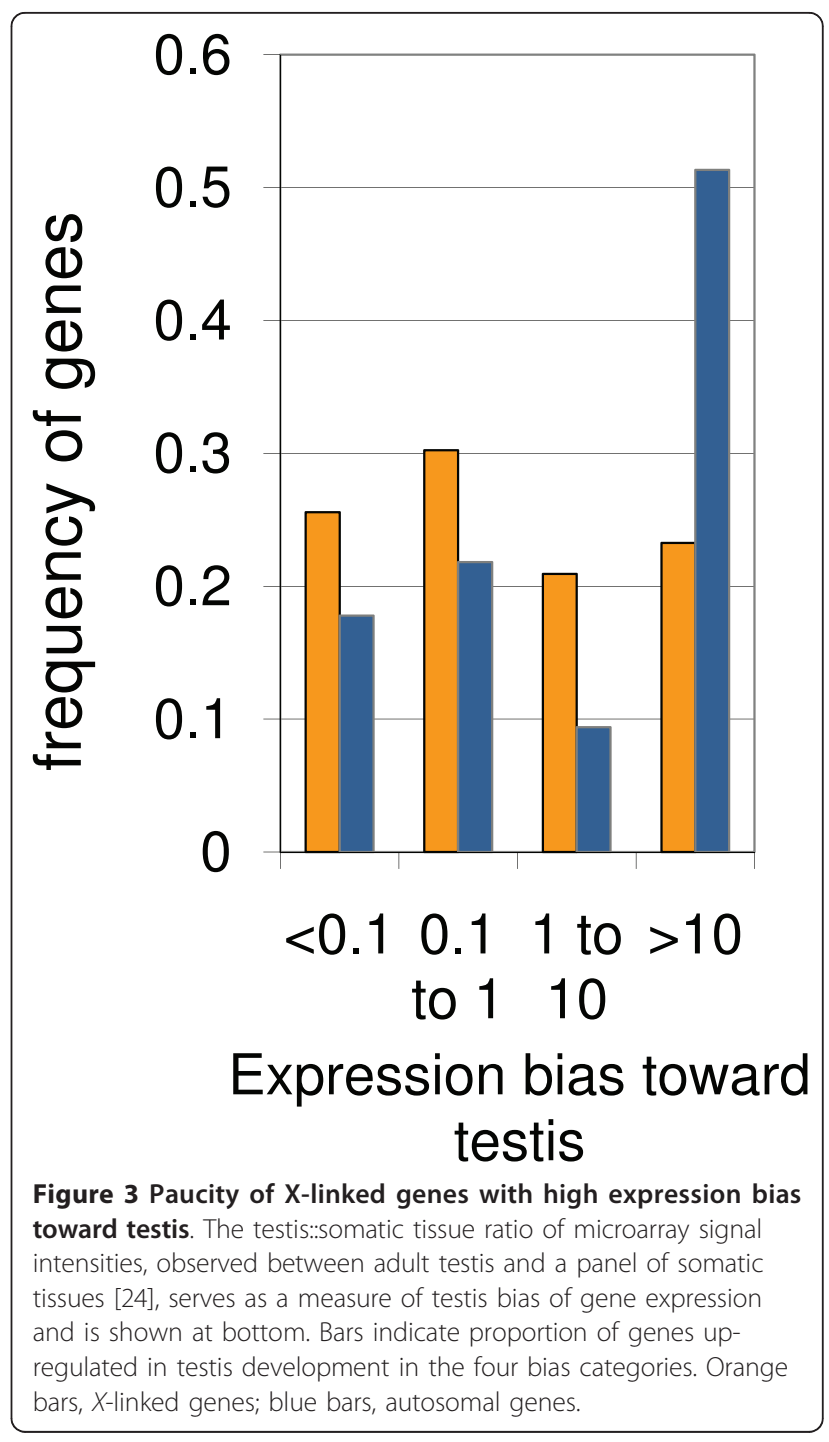

chromosome is not caused by the spermatocyte-specific events. We therefore hypothesized that the underlying mechanisms may be not restricted to the male meiotic germline and instead could operate in diverse tissues, causing broad effects on tissue-biased expression. To test this suggestion, using the published gene expression dataset [27] we identified genes that show expression bias toward midgut, malpigian tubules, accessory gland, salivary gland, head, and ovary, using the same bioinformatics approach as described above for testis. We further determined whether the frequencies of the identified genes on the $X$ chromosome deviated from the genome averages. We observed that the majority of the analyzed genes show the same trends that the testis-biased genes do: they are underrepresented on the $X$ chromosome, and the higher the expression bias the stronger the underrepresentation (Figure 4). One important exception, however, was the ovary-biased gene set for which both trends were reversed. 


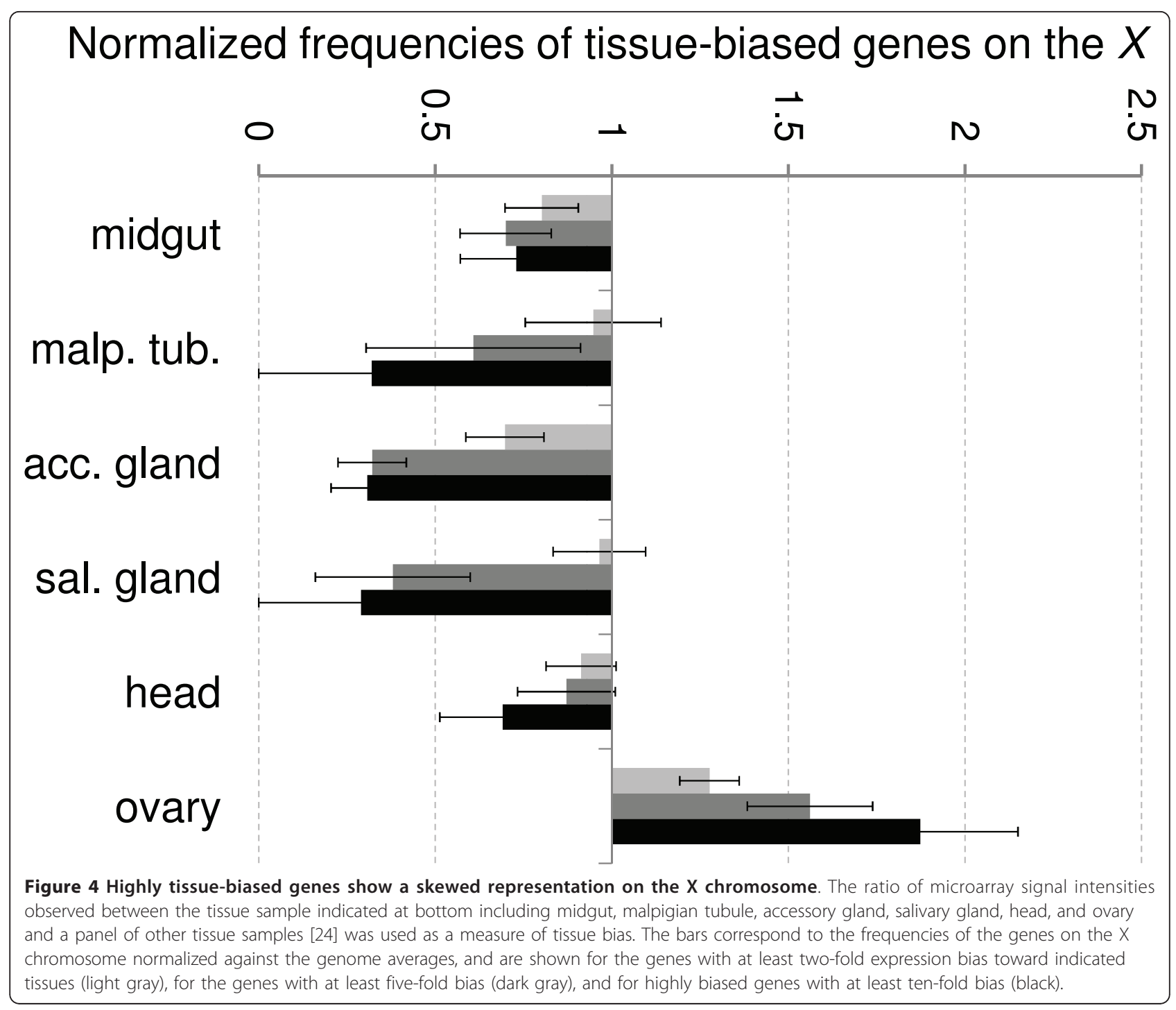

This finding is consistent with previous reports on the overrepresentation of female- and ovary-biased genes on the $X[13,28]$, and indicates that the genes selectively expressed in the ovary are subject to unique selective pressures, probably owing to their female-beneficial sexuallyantagonistic effects $[7,29]$ and/or because of their peculiar regulation, as discussed below.

\section{Genes activated in testis development appear to be repressed in embryos and cultured somatic cells}

Based on our analyses, we suggest that the $X$ chromosome provides an inferior environment for specialized genes with expression highly biased toward particular differentiated cell types. To gain a better understanding of the underlying mechanisms, we analyzed the binding of diverse chromatin proteins to the $X$-linked and autosomal tissue-biased genes. First, we analyzed the correlations between gene expression in testis development and binding of 27 chromatin proteins in embryonic and cultured cells. Changes in gene expression were measured as signal fold changes for every time point in our microarray-based analysis; the earliest analyzed stage (four-day old larvae) served as the reference. Chromatin protein binding was assessed as the fold enrichment in chromatin immunoprecipitation or in DNA adenine methyltransferase identification experiments [30,31]. We have found a correlation that was 'inversed' with respect to the major roles of the analyzed proteins: that is, gene up-regulation in testis development was positively correlated with the binding of silencing-associated proteins in embryos and cultured cells (Figure 5, results for proteins with insignificant correlations are not shown). Specifically, testisup-regulated genes showed prominent associations with histone $\mathrm{H} 1$, histone $\mathrm{H} 3$ trimethylated at $\mathrm{K} 27$, LamDm $_{\mathrm{o}}$, D1, and Polycomb (Pc) and Pc group 


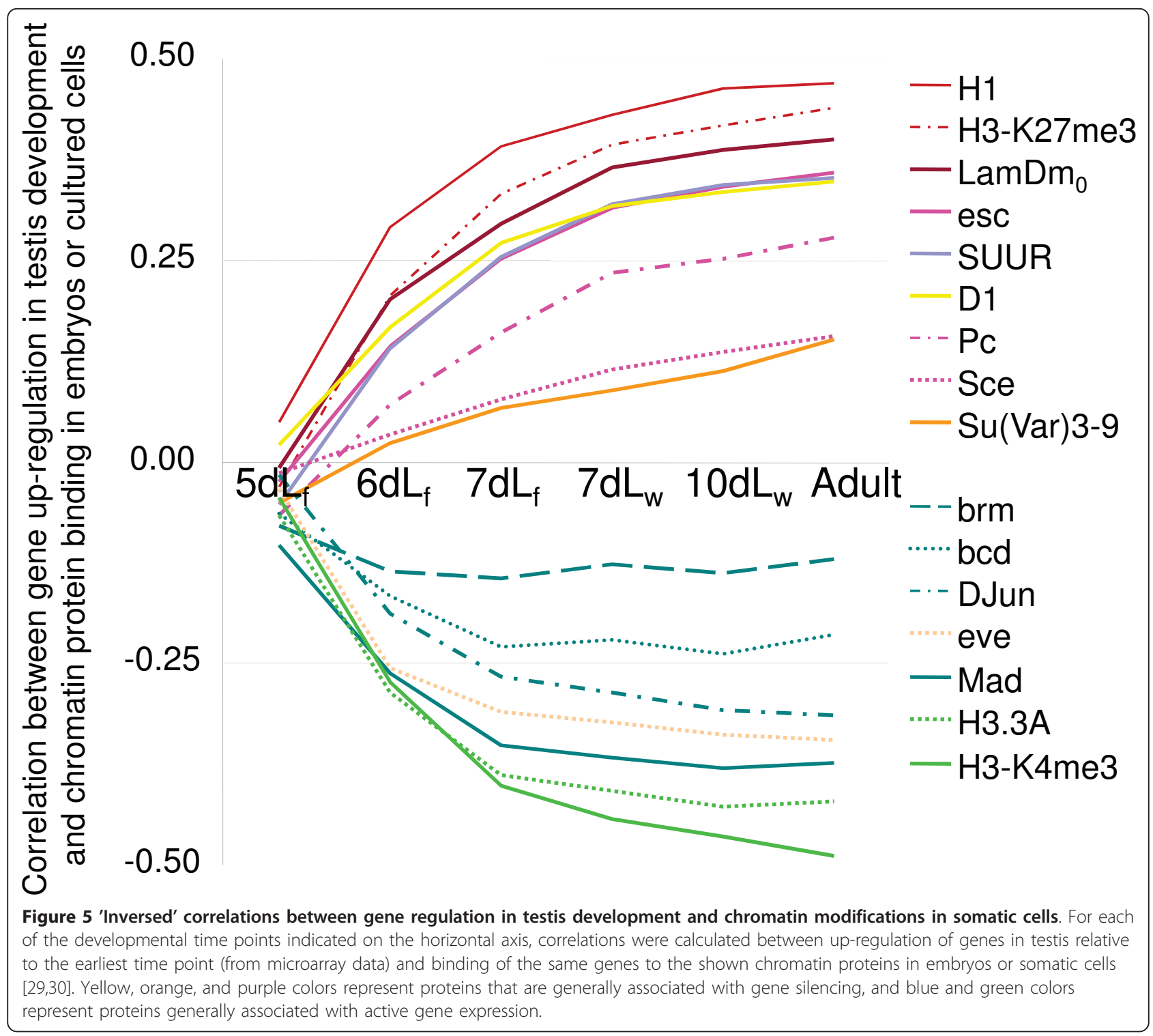

proteins esc and Sce [25,32-38]. In addition, a negative correlation was apparent between genes up-regulated in testis and the binding of gene activation-associated proteins in embryos and cultured cells (these proteins include histone variant $\mathrm{H} 3.3 \mathrm{~A}, \mathrm{H} 3$ trimethylated at $\mathrm{K} 4$, DJun, and bcd) [39-41]. These observations imply that testis-biased expression is regulated by a two-prong 'switch' mechanism comprised of gene activation in testis (most likely, male germline) and gene repression in other tissues and cell types.

Diverse tissue-biased autosomal genes, unlike their $X$ linked counterparts, show strong evidence for repression in embryos and cultured cells

We inquired whether the tissue-biased gene activation generally correlates with binding of repressor proteins in the 'non-target' embryonic and cultured cells, and whether such correlation differs between the $X$ chromosome and autosomes. For this analysis, we calculated the relative enrichment with chromatin proteins for the genes that show more than two-fold expression bias toward testis, midgut, accessory gland, salivary gland, malpigian tubule, and ovary. The tissue expression bias was determined using the same approach as described above for the analysis of tissue-biased gene frequencies on chromosomes. Using arbitrarily-set thresholds to define the categories of bound versus non-bound genes for each of the analyzed chromatin proteins [30,31], we determined the frequencies of bound genes in the sets of tissue-biased genes on the $X$ chromosome and autosomes and normalized them to the frequencies of bound genes in the entire genome. The study included nine 
repressor proteins and eight activators that showed the strongest 'inverse' correlations in previous analysis. The resulting graphs (Figure 6) show whether the $X$-linked (orange bars) or autosomal (blue bars) genes bind repressors or activators in embryos and cultured cells with a frequency different from the genome average (defined as 1.0).

Consistent with the above correlation studies, this analysis showed that autosomal testis-biased genes are enriched with repressors and rarely bind activators in embryos and cultured cells. In the case of the $X$-linked genes this trend is reduced for the vast majority of the analyzed proteins and even reversed for three repressors and five activators (Figure 6). Therefore, the drastic differences in expression status between germline and somatic cells are less common for the testis-biased genes located on the $X$, indicating that the activation/ repression 'switch' is not prominent in the regulation of these genes. Similar observations were made for the majority of genes with an expression bias toward other tissues, with a few exceptions including the malpigian tubule-biased autosomal genes showing enrichment with activators, and the salivary gland-biased $X$-linked genes showing strong enrichment with repressors (Figure 6). In general however, we found that, unlike their autosomal counterparts, the $X$-linked tissue-biased genes are not enriched with repressors and do not lack activators in embryos and cultured cells, implying that the tissuebiased up-regulation of the $X$-linked genes is usually not complemented by their repression in other, 'non-target' cell types. In this respect, ovary-biased genes may provide an example of tissue-biased genes that apparently do not rely on the activation/repression mechanism, regardless of their chromosomal location, because, unlike other tissue-biased genes, they are strongly associated with activators and do not bind repressors in embryonic and cultured cells. This unique feature may facilitate accumulation of the ovary-biased genes on the $X$ chromosome.

\section{Conclusions}

Our findings presented in this study strongly suggest that global inactivation of the $X$ chromosome does not occur in the Drosophila meiotic male germline, and therefore other factors and mechanisms should be invoked to explain the peculiarities of $X$-linked gene expression. A study of gene expression in testis development using microarrays and qRT-PCR, combined with analysis of published in situ hybridization data convincingly show that the majority of $X$-linked genes do not undergo silencing concomitant with meiosis; in fact, many of them are activated in the meiotic germline cells. In agreement with the analysis of sex-biased expression in several Drosophila species [18], we conclude that the possible appearance of $X$-chromosome underexpression in male gonads results from the paucity of $X$-linked testis-biased genes rather than from chromosome-wide gene silencing. Why the $X$ chromosome behaves in meiotic cells in Drosophila unlike in mammals is not clear; perhaps the yet unidentified mechanisms that cause overexpression of $X$-linked genes in the Drosophila germline [18] counteract global silencing of the $X$ chromosome.

Our findings could be consistent with the sexual antagonism driving $X$ inactivation (SAXI) model [10], in which the pressure of sexual selection drives the 'exodus' of male germline-specific genes from the $X$ chromosome causing its inactivation in meiotic cells, with the one exception that no true global $X$ inactivation is seen, just the lack of testis-biased genes on the $X$. However, a recent study indicates that retroposing genes with diverse expression biases all show an affinity toward autosomes [15]; therefore the apparent 'exodus' of male-biased genes from the $X$, previously considered as the major indirect evidence for MSCI in flies [1], is not necessarily driven by sexual selection. Earlier studies did show that not only testis-biased, but also somatic male-biased genes are underrepresented on the $X$ [42], but the possible role of these genes in sexual selection could still be invoked with certain ease owing to their sex bias. Here, we showed that the genes with strong expression bias toward tissues that are not directly related to reproduction, such as salivary glands, midgut, and malpigian tubules, also show paucity on the $X$ chromosome. Taking into account the recent report that somatic gene expression may be regulated by gonads [43], we inquired whether the gonad-dependent genes appreciably contributed to our tissue-biased gene sets. The heaviest representation of gonad-dependent genes [43] was found, not surprisingly, in the testis-biased and ovary-biased gene sets but in both cases it was less than $4 \%$, and less than $2 \%$ of the genes preferentially expressed in salivary glands, midgut, or malpigian tubules appear to be gonad-dependent. Thus, the paucity of tissue-biased genes, and probably the underlying 'exodus' of such genes from the $X$ chromosome, involves genes that have no apparent specific role in reproduction.

A possible clue to the reasons why tissue-biased genes are so scarce on the $X$ is provided by the finding that the genes with the highest bias are affected much more than the genes with modest expression differences (Figure 4B). Based on the analysis of chromatin protein binding to the biased genes, we hypothesize that the high expression bias results from the combined effect of gene activation in the 'target' cells and repression in other cell types. Our analysis indicates that such a mechanism does not operate frequently on the $X$-linked 


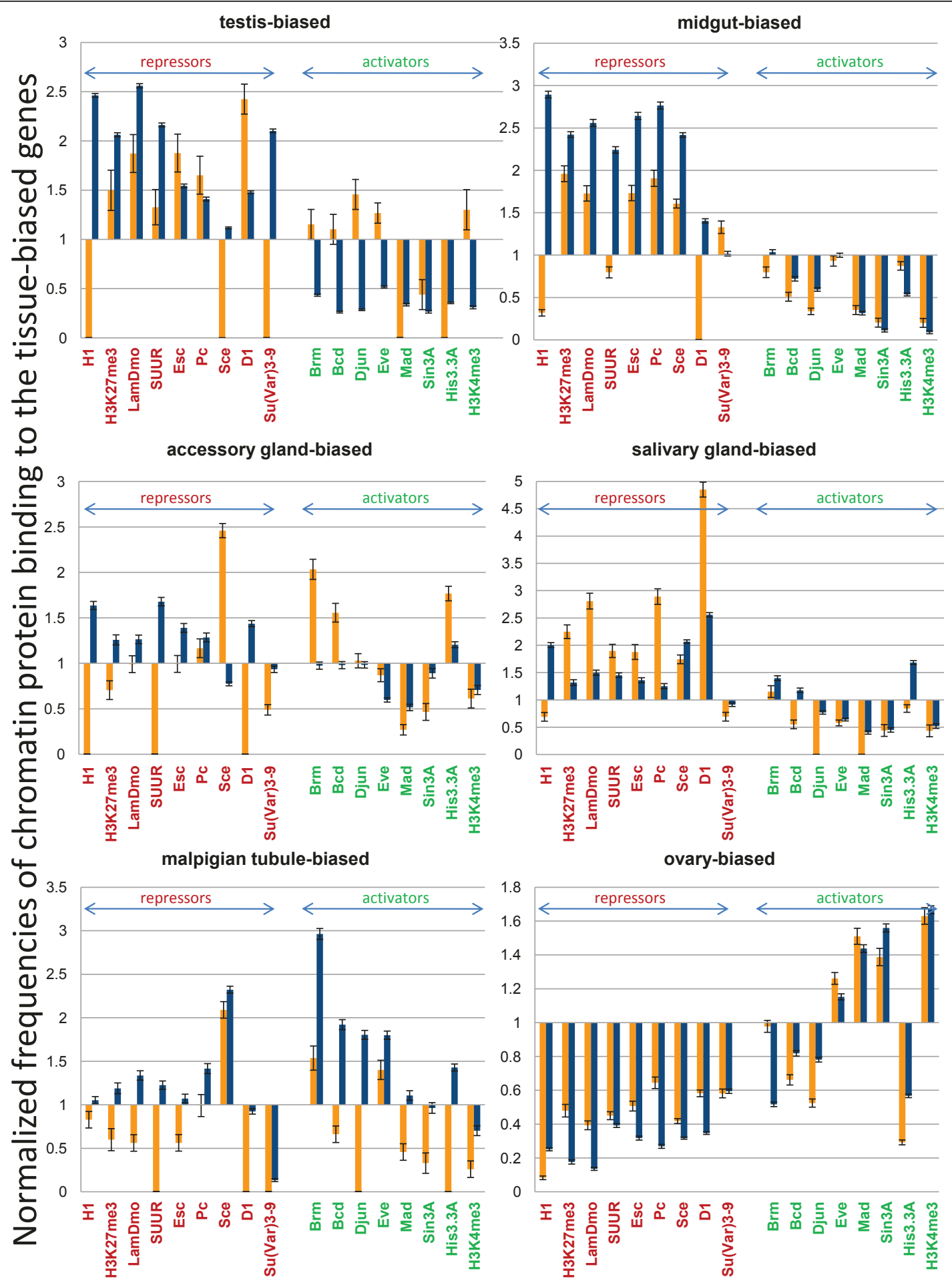

Figure 6 The autosomal and the $X$-linked tissue-biased genes show different patterns of chromatin modifications. The frequencies of binding targets for the proteins indicated at the bottom were calculated among the $X$-linked (orange) and autosomal (blue) tissue-biased genes. The sets of genes showing at least two-fold expression bias toward the indicated tissues (such as testis, midgut, accessory gland, salivary gland, malpigian tubule, and ovary) were generated from the genome-wide expression data [24]. Protein binding genes were defined using chromatin immunoprecipitation and DamID data [29,30] with arbitrarily set thresholds. Bars show relative increase or decrease in binding target frequency within analyzed gene sets as compared to the entire genome. 
tissue-biased genes. This finding presents 'the chicken or the egg' dilemma because we cannot discern whether the weak tissue bias of expression results from not using the activation/repression 'switch' mechanism, or vice versa. Nevertheless, it is possible that the inefficiency of this 'switch' on the $X$ chromosome significantly affects genome evolution. The importance of this influence is illustrated by the unique example of the ovary-biased genes that apparently do not rely on the activation/ repression mechanism (Figure 6) and strongly accumulate on the $X$ chromosome (Figure 4) in perfect agreement with the female-beneficial selection model $[7,29]$.

Of the two components of the activation/repression switch, the repression may be vulnerable to the $X$ chromosome-specific mechanism of dosage compensation that broadly and ubiquitously activates $X$-linked genes in somatic cells [44]. To test this suggestion, we analyzed binding of the testis-up-regulated genes with the key dosage compensation component msl-2 using published datasets $[45,46]$ expecting to find that the highly testis-biased genes do not bind msl-2. We found no correlation between testis bias of expression and msl-2 binding. However, dosage compensation machinery can regulate genes at a distance from the primary binding sites [47] and a recent study did show that the genes with the highest male bias of expression tend to localize outside the compensated regions [48]. We propose, therefore, that the H4-K16 acetylation of the $X$ chromosome by the dosage compensation complex interferes with highly tissue-biased gene expression by counteracting repression in the 'non-target' cell types. This may present a significant problem if the gene product is toxic to diverse cells (which for example could be expected for many sperm differentiation genes), and could result in a strong selective pressure toward relocation of the highly tissue-biased genes to the autosomes.

\section{Methods}

\section{Fly culture and dissection}

Wild type (Oregon) larvae were grown on yeastmolasses media at $18^{\circ} \mathrm{C}$. At the specified time points larvae were collected, and testes harvested using manual dissection. Further analyses utilized samples pooled from at least 30 larvae.

\section{Quantitative RT-PCR}

Total RNA was isolated from dissected testes with Trizol reagent (Invitrogen, Carlsbad, CA, USA), and cDNA synthesized from $1 \mu \mathrm{g}$ RNA samples using oligo(dT) primer and SuperScript II reverse transcriptase (Invitrogen). Real-time PCR was performed in the iCycler iQ5 instrument (Bio-Rad, Hercules, CA, USA) using SYBR Green chemistry; rp49 transcripts served as template loading reference.

\section{Microarray probes}

Total RNA was isolated from testes with Trizol reagent (Invitrogen), and further purified with the RNeasy kit (Qiagen, Hilden, North Rhine-Westphalia, Germany). Poly(A)+ RNA was selectively amplified from 0.5 to 1 $\mu \mathrm{g}$ total RNA samples with the BD SMART cDNA Synthesis kit and the BD Advantage 2 PCR Enzyme System (Clontech, Mountain View, CA, USA). Amplified cDNAs were cleaned with the Wizard SV Gel kit (Promega, Madison, WI, USA). cDNA samples of 3.5 to 4.5 $\mu \mathrm{g}$ were labeled with the ULYSIS Alexa Fluor 546 or Alexa Fluor 647 (Molecular Probes, Eugene, OR, USA) dyes according to the manufacturer's protocol, and used for competitive hybridization with microarrays.

\section{Hybridization of samples to microarrays}

The Drosophila oligonucleotide microarray set (QiagenOperon) was printed on aminosilane-coated slides at Tufts-New England Medical Center Expression Array Core facility (TEAC). The set contains 14,593 70-mer oligonucleotides representing 13,664 genes which cover most of genes in release 3.2 of the Drosophila genome (The FlyBase Consortium, 2003). The probe sequences from the Operon set were re-mapped to Drosophila genome release 5.1 using BLAST, and probes with multiple hits or ambiguous mapping were excluded from subsequent analysis. Additional information on the Drosophila oligonucleotide set can be found at the manufacturer's website http://www.operon.com. Details of the array design can be found at ArrayExpress (array design Operon AROS D. melanogaster v.1.1). Labeled pairs of samples were hybridized in $1 \times$ hybridization buffer (GE Healthcare, Little Chalfont, Buckinghamshire, UK), 20\% formamide, and $0.025 \%$ each of Ficoll, polyvinylpyrrolidone, Na pyrophosphate, and heparin. Samples were incubated with microarrays for 44 hours at $37^{\circ} \mathrm{C}$. After hybridization slides were washed, dried by centrifugation, and scanned on the ScanArray 4000 scanner (PerkinElmer, Waltham, MA, USA).

\section{Data acquisition and analysis}

Fluorescence intensities of individual spots were acquired from the array images with ImaGene software (BioDiscovery). Subsequent normalization and statistical analysis were performed with package limma [49,50], part of the BioConductor project [51]. Normalization of the data is described in Normalization protocol associated with ArrayExpress submission E-MEXP-1980 of the microarray experiment described in this manuscript. At least three hybridization replicates passing quality control were performed per experimental condition. Data were analyzed using the linear modeling approach, and Bayes moderated t test was used to determine differential expression between 
each time point and the fourth day (the earliest time point). q values for individual contrasts represent FDR for multiple testing [52]. q $<0.1$ was chosen as the threshold for differential expression unless specified differently.

Hypergeometric analysis of the representation of differentially expressed genes on Drosophila chromosomes was performed with the web-based tool GeneMerge (http://genemerge.cbcb.umd.edu/[53]). Significance of the differences in bin content for different chromosome arms (see Figure 4 and related text in Results section) was assessed with Fisher's exact test as implemented in $\mathrm{R}$, and correlations between the gene expression and protein binding data were calculated with two-sided Pearson's method, also as implemented in R [54]. The significance of Fisher's and Pearson's tests was adjusted for multiple testing according to Bonferroni method within corresponding data series.

\section{Abbreviations}

MSCl: meiotic sex chromosome inactivation; qRT-PCR: reverse transcription followed with quantitative polymerase chain reaction analysis; FDR: false discovery rate.

\section{Acknowledgements}

This work was supported by National Institutes of Health grant GM61549.

\section{Author details}

'Department of Anatomy and Cellular Biology, Tufts University School of Medicine, Boston, USA. ²Department of Biochemistry and Molecular Biology, University of Maryland School of Medicine, Baltimore, USA.

\section{Authors' contributions}

DIN designed research; LMM performed research; LMM and DIN analyzed data; and DIN wrote the paper. All authors read and approved the final manuscript.

\section{Received: 7 April 2011 Accepted: 4 May 2011 Published: 4 May 2011}

\section{References}

1. Betrán $\mathrm{E}$, Thornton $\mathrm{K}$, Long $\mathrm{M}$ : Retroposed new genes out of the $X$ in Drosophila. Genome Res 2002, 12:1854-1859.

2. Emerson JJ, Kaessmann H, Betrán E, Long M: Extensive gene traffic on the mammalian X chromosome. Science 2004, 303:537-540.

3. Vinckenbosch N, Dupanloup I, Kaessmann H: Evolutionary fate of retroposed gene copies in the human genome. Proc Natl Acad Sci USA 2006, 103:3220-3225.

4. Potrzebowski $L$, Vinckenbosch $N$, Marques AC, Chalmel F, Jégou B, Kaessmann $\mathrm{H}$ : Chromosomal gene movements reflect the recent origin and biology of therian sex chromosomes. PLoS Biol 2008, 6:e80.

5. Khil PP, Smirnova NA, Romanienko PJ, Camerini-Otero RD: The mouse $X$ chromosome is enriched for sex-biased genes not subject to selection by meiotic sex chromosome inactivation. Nat Genet 2004, 36:642-646.

6. Mueller JL, Mahadevaiah SK, Park PJ, Warburton PE, Page DC, Turner JM: The mouse $X$ chromosome is enriched for multicopy testis genes showing postmeiotic expression. Nat Genet 2008, 40:794-799.

7. Rice WR: Sex chromosomes and the evolution of sexual dimorphism. Evolution 1984, 38:735-742.

8. White-Cooper $\mathrm{H}$ : Molecular mechanisms of gene regulation during Drosophila spermatogenesis. Reproduction 2010, 139:11-21.

9. Lifschytz $\mathrm{E}$, Lindsley $\mathrm{DL}$ : The role of $X$-chromosome inactivation during spermatogenesis. Proc Nat Acad Sci USA 1972, 69:182-186.

10. Wu C $-1, X u E Y$ : Sexual antagonism and $X$ inactivation-the SAXI hypothesis. Trends Genet 2003, 19:243-247.
11. Gurbich TA, Bachtrog D: Gene content evolution on the $X$ chromosome Curr Opin Genet Dev 2008, 18:493-498.

12. Boutanaev AM, Kalmykova Al, Shevelyov YY, Nurminsky DI: Large clusters of co-expressed genes in the Drosophila genome. Nature 2002, 420:666-669.

13. Parisi $M$, Nuttall $R$, Naiman D, Bouffard G, Malley J, Andrews J, Eastman S, Oliver B: Paucity of genes on the Drosophila X chromosome showing male-biased expression. Science 2003, 299:697-700.

14. Sturgill D, Zhang $Y$, Parisi M, Oliver B: Demasculinization of $X$ chromosomes in the Drosophila genus. Nature 2007, 450:238-241.

15. Metta M, Schlötterer C: Non-random genomic integration - an intrinsic property of retrogenes in Drosophila? BMC Evol Biol 2010, 10:114.

16. Hense W, Baines JF, Parsch J: $X$ chromosome inactivation during Drosophila spermatogenesis. PLoS Biol 2007, 5:e273.

17. Vibranovski MD, Lopes HF, Karr TL, Long M: Stage-specific expression profiling of Drosophila spermatogenesis suggests that meiotic sex chromosome inactivation drives genomic relocation of testis-expressed genes. PLoS Genet 2009, 5:e1000731.

18. Gupta V, Parisi M, Sturgill D, Nuttall R, Doctolero M, Dudko OK, Malley JD, Eastman PS, Oliver B: Global analysis of X-chromosome dosage compensation. J Biol 2006, 5:3.

19. Olivieri G, Olivieri A: Autoradiographic study of nucleic acid synthesis during spermatogenesis in Drosophila melanogaster. Mutat Res 1965, 2:366-380

20. Lindsey DL, Tokuyasu KT: Spermatogenesis. In The Genetics and Biology of Drosophila. Volume 2. Edited by: Ashbumer M, Wright TRF. London: Academic Press; 1980:226-294.

21. Zhao J, Klyne G, Benson E, Gudmannsdottir E, White-Cooper H, Shotton D: FlyTED: the Drosophila Testis Gene Expression Database. Nucl Acids Res 2010, 38:D710-D715.

22. Nurminsky DI, Nurminskaya MV, De Aguiar D, Hartl DL: Selective sweep of a newly evolved sperm-specific gene in Drosophila. Nature 1998, 396:572-575.

23. Blümer N, Schreiter K, Hempel L, Santel A, Hollmann M, Schäfer MA, Renkawitz-Pohl R: A new translational repression element and unusual transcriptional control regulate expression of don juan during Drosophila spermatogenesis. Mech Dev 2002, 110:97-112.

24. Hwa JJ, Hiller MA, Fuller MT, Santel A: Differential expression of the Drosophila mitofusin genes fuzzy onions ( $f z 0)$ and dmfn. Mech Dev 2002, 116:213-216.

25. Michiels F, Gasch A, Kaltschmidt B, Renkawitz-Pohl R: A 14 bp promoter element directs the testis specificity of the Drosophila beta 2 tubulin gene. EMBO J 1989, 8:1559-1565.

26. Shevelyov YY, Lavrov SA, Mikhaylova LM, Nurminsky ID, Kulathinal RJ, Egorova KS, Rozovsky YM, Nurminsky DI: The B-type lamin is required for somatic repression of testis-specific gene clusters. Proc Natl Acad Sci USA 2009, 106:3282-3287.

27. Chintapalli VR, Wang J, Dow JA: Using FlyAtlas to identify better Drosophila melanogaster models of human disease. Nat Genet 2007, 39:715-720.

28. Ranz JM, Castillo-Davis Cl, Meiklejohn CD, Hartl DL: Sex-dependent gene expression and evolution of the Drosophila transcriptome. Science 2003, 300:1742-1745.

29. Vicoso B, Charlesworth B: Evolution on the $X$ chromosome: unusual patterns and processes. Nat Rev Genet 2006, 7:645-653.

30. Schwartz YB, Kahn TG, Nix DA, Li XY, Bourgon R, Biggin M, Pirrotta V: Genome-wide analysis of Polycomb targets in Drosophila melanogaster Nat Genet 2006, 38:700-705.

31. de Wit E, Braunschweig U, Greil F, Bussemaker HJ, van Steensel B: Global chromatin domain organization of the Drosophila genome. PLoS Genet 2008, 24:e1000045.

32. Ni JQ, Liu LP, Hess D, Rietdorf J, Sun FL: Drosophila ribosomal proteins are associated with linker histone $\mathrm{H} 1$ and suppress gene transcription. Genes Dev 2006, 20:1959-1973.

33. Kim K, Choi J, Heo K, Kim H, Levens D, Kohno K, Johnson EM, Brock HW, An W: Isolation and characterization of a novel $\mathrm{H} 1.2$ complex that acts as a repressor of p53-mediated transcription. J Biol Chem 2008, 283:9113-9126.

34. Schones DE, Zhao K: Genome-wide approaches to studying chromatin modifications. Nat Rev Genet 2008, 9:179-191.

35. Simon JA, Tamkun JW: Programming off and on states in chromatin: mechanisms of Polycomb and trithorax group complexes. Curr Opin Genet Dev 2002, 12:210-218. 
36. Czermin B, Melfi R, McCabe D, Seitz V, Imhof A, Pirrotta V: Drosophila enhancer of Zeste/ESC complexes have a histone $\mathrm{H} 3$ methyltransferase activity that marks chromosomal Polycomb sites. Cell 2002, 111:185-196.

37. Fritsch C, Beuchle D, Muller J: Molecular and genetic analysis of the Polycomb group gene Sex combs extra/Ring in Drosophila. Mech Dev 2003, 120:949-954

38. Aulner N, Monod C, Mandicourt G, Jullien D, Cuvier O, Sall A, Janssen S, Laemmli UK, Kas E: The AT-hook protein D1 is essential for Drosophila melanogaster development and is implicated in position-effect variegation. Molec Cell Biol 2002, 22:1218-1232.

39. Ma J: Crossing the line between activation and repression. Trends Genet 2005, 21:54-59.

40. Mito Y, Henikoff JG, Henikoff S: Genome-scale profiling of histone H3.3 replacement patterns. Nat Genet 2005, 37:1090-1097.

41. Kockel L, Homsy JG, Bohmann D: Drosophila AP-1: lessons from an invertebrate. Oncogene 2001, 20:2347-2364.

42. Sturgill D, Zhang Y, Parisi M, Oliver B: Demasculinization of $X$ chromosomes in the Drosophila genus. Nature 2007, 450:238-241.

43. Parisi MJ, Gupta V, Sturgill D, Warren JT, Jallon JM, Malone JH, Zhang Y, Gilbert LI, Oliver B: Germline-dependent gene expression in distant nongonadal somatic tissues of Drosophila. BMC Genomics 2010, 11:346.

44. Akhtar A, Becker P: Activation of transcription through histone $\mathrm{H} 4$ acetylation by MOF, an acetyltransferase essential for dosage compensation in Drosophila. Mol Cell 2000, 5:367-375.

45. Alekseyenko AA, Larschan E, Lai WR, Park PJ, Kuroda MI: High-resolution ChIP-chip analysis reveals that the Drosophila MSL complex selectively identifies active genes on the male $X$ chromosome. Genes Dev 2006, 20:848-857.

46. Gilfillan GD, Straub T, de Wit E, Greil F, Lamm R, van Steensel B, Becker PB: Chromosome-wide gene-specific targeting of the Drosophila dosage compensation complex. Genes Dev 2006, 20:858-870.

47. Larschan E, Alekseyenko AA, Gortchakov AA, Peng S, Li B, Yang P, Workman JL, Park PJ, Kuroda MI: MSL complex is attracted to genes marked by $\mathrm{H} 3 \mathrm{~K} 36$ trimethylation using a sequence-independent mechanism. Mol Cell 2007, 28:121-133.

48. Bachtrog D, Toda NR, Lockton S: Dosage compensation and demasculinization of X chromosomes in Drosophila. Curr Biol 2010, 20:1476-1481

49. Smyth GK: Limma: linear models for microarray data. In Bioinformatics and Computational Biology Solutions using $R$ and Bioconductor. Edited by: Gentleman R, Carey V, Dudoit S, Irizarry R, Huber W. New York: Springer; 2005:397-420.

50. Smyth G K, Yang Y-H, Speed TP: Statistical issues in microarray data analysis. Methods Mol Biol 2003, 224:111-136.

51. Gentleman RC, Carey VJ, Bates DM, Bolstad B, Dettling M, Dudoit S, Ellis B, Gautier L, Ge Y, Gentry J, Hornik K, Hothorn T, Huber W, lacus S, Irizarry R, Leisch F, Li C, Maechler M, Rossini AJ, Sawitzki G, Smith C, Smyth G, Tierney L, Yang JY, Zhang J: Bioconductor: open software development for computational biology and bioinformatics. Genome Biol 2004, 5:R80.

52. Benjamini $Y$, Hochberg $Y$ : Controlling the false discovery rate: a practical and powerful approach to multiple testing. J R Statist Soc B 1995 57:289-300.

53. Castillo-Davis Cl, Hartl DL: GeneMerge: post-genomic analysis, data mining, and hypothesis testing. Bioinformatics 2003, 19:891-892.

54. R Development Core Team: R: A Language and Environment for Statistical Computing. Vienna: R Foundation for Statistical Computing 2006 [http://www.R-project.org].

doi:10.1186/1741-7007-9-29

Cite this article as: Mikhaylova and Nurminsky: Lack of global meiotic sex chromosome inactivation, and paucity of tissue-specific gene expression on the Drosophila X chromosome. BMC Biology 2011 9:29.

\section{Submit your next manuscript to BioMed Central and take full advantage of:}

- Convenient online submission

- Thorough peer review

- No space constraints or color figure charges

- Immediate publication on acceptance

- Inclusion in PubMed, CAS, Scopus and Google Scholar

- Research which is freely available for redistribution

Submit your manuscript at www.biomedcentral.com/submit
C Biomed Central 